\title{
ESTILOS DE APRENDIZAGEM E TECNOLOGIAS DA INFORMAÇÃO E COMUNICAÇÃO NO CURSO DE DIREITO' ${ }^{1}$
}

\author{
ESTILOS DE APRENDIZAJE Y TECNOLOGÍAS DE INFORMACIÓN Y \\ COMUNICACIÓN EN EL CURSO DE DERECHO
}

\section{LEARNING STYLES AND THE USAGE OF THE INFORMATION AND COMMUNICATION TECHNOLOGIES IN LAW SCHOOL}

\author{
Helena Brandão VIANA ${ }^{2}$ \\ Meira Lúcia RAMOS ${ }^{3}$ \\ Rodrigo Hipólito ROZA ${ }^{4}$
}

RESUMO: Esta pesquisa teve como objetivos identificar os estilos de aprendizagem no uso da tecnologia em alunos e docentes de curso de Direito. Foi utilizada a Escala de Estilos de Aprendizagem em situações de uso de tecnologia de Roza (2017), que foi aplicada em 444 estudantes do curso de Direito de duas instituições particulares, bem como em 23 docentes desses cursos. Para análise de dados, foi utilizada estatística descritiva e análise multivariada da variância (MANOVA) para verificar as possíveis influências do gênero e da fase do estudante sobre os estilos dos alunos. Os resultados mostraram uma predominância do estilo teórico entre todos os estudantes. Entre os professores predominou o estilo pragmático. Embora o estilo teórico tenha sido predominante, os outros estilos foram também contemplados. Portanto, visualiza-se a importância da utilização de diferentes ferramentas tecnológicas e estratégias educacionais que contemplem os diferentes estilos de aprendizagem dos alunos.

PALAVRAS-CHAVE: Estilos de aprendizagem. Direito. Tecnologia educacional.

RESUMEN: Esta investigación tuvo como objetivo identificar los estilos de aprendizaje en el uso de la tecnología en alumnos y profesores de cursos de Derecho. Se utilizó la Escala de Estilos de Aprendizaje en situaciones de uso de tecnología por Roza (2017), que se aplicó a 444 estudiantes del curso de Derecho en dos instituciones privadas, así como a 23 profesores de estos cursos. Para el análisis de datos, se utilizaron estadísticas descriptivas y análisis de varianza multivariante (MANOVA) para verificar las posibles influencias del género y la fase del alumno en los estilos de los estudiantes. Los resultados mostraron un predominio del estilo teórico entre todos los estudiantes. Entre los profesores, prevaleció el estilo pragmático. Aunque predominaba el estilo teórico, también se contemplaron los otros estilos.

\footnotetext{
${ }^{1}$ Pesquisa registrada no CEP - CAEE 66631317.0.0000.5377

${ }^{2}$ Centro Universitário Adventista São Paulo (UNASP), Engenheiro Coelho - SP - Brasil. Professora Permanente no Mestrado Profissional em Educação. Doutorado em Educação Física (UNICAMP). ORCID: https://orcid.org/ 0000-0002-2018-202X. E-mail: hbviana2@gmail.com

${ }^{3}$ UNIMOGI, Mogi Guaçu - SP - Brasil. Professora no curso de Direito. Mestrado Profissional em Educação (UNASP). ORCID: https://orcid.org/0000-0001-8948-0837. E-mail: meiralu@yahoo.com

${ }^{4}$ Pontifícia Universidade Católica de Campinas (PUC-CAMPINAS), Campinas - SP - Brasil. Professor do Centro de Economia e Administração. Pós-Doutorado em Psicologia (USF). ORCID: https://orcid.org/00000002-7162-4908. E-mail: rodrigo.roza@gmail.com
} 
Por lo tanto, se puede ver la importancia de utilizar diferentes herramientas tecnológicas y estrategias educativas que contemplen los diferentes estilos de aprendizaje de los estudiantes.

PALABRAS CLAVE: Estilos de aprendizaje. Derecho. Tecnología educacional.

ABSTRACT: This research aimed to identify the learning styles with the usage of technology on students and professors of law school. The Learning Styles Scale was used in situations of technology use by Roza (2017), which was applied to 444 Law School students in two private institutions as well as to 23 of their professors. As for the data analysis, descriptive statistics, and multivariate analysis of variance (MANOVA) were used to verify the possible influences of gender and student phase on students' styles. The results showed a predominance of the theoretical style among all students. Among the professors, the pragmatic style prevailed. Although the theoretical style was predominant, the other styles were also contemplated. Therefore, the importance of using different technological tools and educational strategies that contemplate the different learning styles of students is visualized.

KEYWORDS: Learning styles. Law. Educational technology.

\section{Introdução}

Uma nova forma de organização social, intitulada sociedade da informação, surgiu na segunda metade do século XX, proveniente da revolução tecnológica (ROZA, 2017). As mudanças nessa nova sociedade ocorreram no cenário econômico, social e humano, já que o desenvolvimento das tecnologias digitais influenciou não só nas relações sociais, mas também na produção, industrialização e no ambiente educacional, permitindo às próprias crianças o acesso às informações e ao conhecimento do mundo, através das mídias. (RIEDNER; PISCHETOLA, 2016).

Nessa nova sociedade, também denominada de sociedade do conhecimento ou sociedade em rede, não há barreiras no que diz respeito ao tempo e ao espaço que impeçam as pessoas de se comunicarem. Trata-se de uma nova era que possibilita diversas formas de aprender, deixando de ser o espaço físico da escola como único local para a construção do conhecimento (COUTINHO; LISBÔA, 2011).

Essa nova sociedade também impôs grandes desafios à escola, já que esta tem de ser capaz de desenvolver competências nos alunos para conviver num mundo global, que valoriza um ser criativo e capaz de apresentar soluções inovadoras para os problemas futuros (COUTINHO; LISBÔA, 2011). Os alunos, ao contrário das gerações passadas, pensam e processam de forma diferente as informações, não podendo tal fato ser ignorado pelo professor, nem tampouco suas implicações para o processo de ensino aprendizagem (SILVA, 
2017). Persistir em um modelo pedagógico baseado em aulas expositivas e com pouca criatividade pode desestimular os alunos no decorrer de um curso (GOES et al., 2014).

Também, a partir da segunda metade do século XX, com base em um novo conceito de aprendizagem, centrado no papel ativo do aluno e contrário às metodologias tradicionais, diversos autores vêm se ocupando do estudo dos estilos de aprendizagem (SILVA, 2011). Os estilos de aprendizagem são as preferências de cada indivíduo, fazendo parte de suas características pessoais e influenciando na forma de aprender o conteúdo (AMARAL; BARROS, 2007). Segundo Gonçalves et al. (2016), estilo de aprendizagem é a forma de absorver, processar e reter a informação. Da mesma forma que os estilos de aprendizagem, os estilos cognitivos também vem sendo objeto de estudos, mas, devido à inexistência de um conceito comum entre os autores a respeito deles, a literatura tem apresentado diferentes definições desses construtos.

Para tentar contribuir com as pesquisas realizadas sobre estilos no uso da tecnologia no processo de ensino e aprendizagem, esta pesquisa teve como objetivo identificar os estilos de aprendizagem no uso da tecnologia para o aprendizado em alunos e docentes do curso de Direito, bem como as influências do gênero e fase do estudante sobre os estilos dos alunos. Como objetivo específico, este trabalho apresentou os pressupostos teóricos dos estilos no uso da tecnologia.

\section{A tecnologia na educação}

A expressão tecnologia da informação pode ser definida como toda tecnologia utilizada no tratamento da informação, abrangendo diversos recursos, dentre eles, a Internet, os computadores, os tablets e os smartphones, aplicativos, sistemas de telecomunicações. (ROZA, 2017). As tecnologias digitais da informação e comunicação (TDIC), presentes cada vez mais em nossa sociedade e em quase todas as camadas sociais, permitem que seus usuários se comuniquem ainda que estando em diferentes lugares. A forma como as pessoas se comunicam foi modificada, sendo quase impossível não verificar alguém acessando a qualquer hora e lugar seu aparelho celular para se comunicar (SILVA, 2017).

A quantidade de informações à disposição dos indivíduos na atual sociedade requer do cidadão o desenvolvimento de capacidades para selecionar aquelas mais relevantes, descartando as desnecessárias, o que exige o desenvolvimento de capacidades para relacionar, analisar, sintetizar e avaliar. Para isso, a escola pode contribuir com alternativas, como o uso 
da informática, para que não haja repetição das metodologias antigas, como a cópia, a reprodução e a memorização (LÂNGARO, 2003).

Com a nova sociedade do conhecimento, o perfil das salas de aula mudou, já que estão repletas de alunos com sede de informação e rapidez na seleção de novos interesses. Essas mudanças da sociedade influenciaram também nos índices de atenção dos alunos, gerando neles a falta de concentração em razão da quantidade compartilhada de dados. (CINTRA; CAMURÇA; REIS, 2017).

As TDIC têm se mostrado como importantes aliadas ao ensino, já que são recursos que não somente estimulam os sentidos, mas proporcionam o compartilhamento de informações e permitem novas formas de comunicação, de relacionamento, de construção do conhecimento, abrindo novas possibilidades pedagógicas (SOUZA, 2015; KENSKI, 2003; PÚBIO JR., 2018).

Algumas vantagens têm impulsionado o uso das tecnologias no campo educacional: facilita a compreensão do conteúdo; respeita o tempo de aprendizagem do discente; permite ao aluno treinar quantas vezes forem necessárias; possibilita o feedback. (GOES et al., 2014).

As TDIC criaram espaços de interação e de construção do conhecimento, bastando alguém estar conectado em rede para trocar informações, ideais e conhecimentos (LEMOS; AMARAL; OLIVEIRA, 2015). Até mesmo na educação inclusiva, elas têm-se mostrado como instrumentos favorecedores da acessibilidade, da equiparação de oportunidades e da inclusão de alunos com necessidades especiais (SOUZA, 2015).

Com o uso das TDIC no processo de ensino-aprendizagem, o saber deixa de estar centrado na figura do professor, já que este deixa de ser observado pelos alunos para ser tornar observador destes, buscando, assim, as potencialidades de cada indivíduo. (SILVA et al., 2010). Ainda, o aluno deve ser visto pelo ensino como um sujeito ativo, com capacidade para determinar sua autoaprendizagem, favorecendo, dentre outras coisas, o pensamento reflexivo e a solução de hipóteses, a partir de estratégias participativas e interativas (GOES et al., 2014).

$\mathrm{O}$ uso das tecnologias no ensino é reconhecido mundialmente como benéfico para o processo inovador dos ambientes de aprendizagem, já que contribui para a formação de um pensamento crítico, a tomada de decisões complexas, a resolução de problemas, o trabalho em equipe, e se pauta na participação ativa dos alunos, desenvolvendo sua autonomia e criticidade (SALVADOR et al., 2015).

Silveira, Novello e Laurino (2018) acreditam que o uso das tecnologias digitais no ensino irá proporcionar aos sujeitos diversas possibilidades de construção de saberes, de troca 
e de construção de novos conhecimentos, além do desenvolvimento de atividades interativas. Entretanto, tem sido enfatizada a importância de ser superada a visão equivocada de que o uso da tecnologia no ensino se encerra em si, uma vez que não é uma ferramenta autossuficiente, sendo incapaz de solucionar todos os problemas do ensino a sua aplicação pura e simples. Referindo-se a um ensino de enfermagem inovador, Salvador et al. (2015) enfatizam que, além do uso das ferramentas tecnológicas nesse processo ensino-aprendizagem, são instituídos os seguintes desafios: a adequada preparação do docente, a transformação da interação professor-aluno, mudanças nas estruturas das instituições educacionais. Assim, segundo esses autores (SALVADOR et al., 2015), a abordagem pedagógica do docente no uso das tecnologias no ensino é mais importante do que a própria tecnologia em si, já que tais ferramentas tecnológicas permitem práticas de ensino em que há a coparticipação entre alunos e professores, acompanhada de interatividade e criatividade.

As universidades, nos últimos tempos, vêm passando por diversas transformações, permitindo que os processos de organização estrutural acadêmica incluam como temas a utilização das ferramentas tecnológicas no processo pedagógico e na formação do professor, o operar através das diferentes ferramentas digitais, objetivando atender as demandas atuais dos indivíduos e da sociedade, possibilitando a construção de uma nova cultura. (SILVEIRA; NOVELLO; LAURINO, 2018).

\section{Procedimentos metodológicos}

Na pesquisa de campo foi utilizada a ESCALA DE ESTILOS DE APRENDIZAGEM EM SITUAÇÕES DE USO DE TECNOLOGIA, de Roza (2017). A escala foi aplicada em alunos dos cursos de Direito, em duas instituições, e em professores destes cursos. Esse instrumento foi construído a partir dos estudos sobre estilos de aprendizagem abordados, principalmente por Alonso, Galego e Honey (1997), Kolb (1984) e Mumford e Honey (1992).

Participaram da pesquisa 444 universitários do curso de Direito, de duas universidades privadas, localizadas no interior de São Paulo (denominadas nesta pesquisa de Instituição 1 e 2), sendo 186 homens e 258 mulheres, com a média de idade de 28.98 anos. As características detalhadas da amostra de estudantes encontram-se na Tabela 1. 
Tabela 1 - Características dos estudantes

\begin{tabular}{|c|c|c|c|c|c|c|c|c|}
\hline \multicolumn{2}{|c|}{ semestres } & \multicolumn{3}{|c|}{ sujeitos } & \multicolumn{4}{|c|}{ idade } \\
\hline Instituição1 & Instituição 2 & $\begin{array}{c}n \\
\text { total }\end{array}$ & homens & mulheres & média & dp & mínimo & máxima \\
\hline \multirow[t]{2}{*}{$1^{\circ}$ semestre } & & 50 & 17 & 33 & 26.8 & 7.95 & 18 & 47 \\
\hline & $1^{\circ}$ semestre & 90 & 34 & 56 & 21.42 & 5.68 & 18 & 36 \\
\hline \multirow[t]{2}{*}{$2^{\circ}$ semestre } & & 13 & 7 & 6 & 31 & 9.18 & 20 & 52 \\
\hline & $2^{\circ}$ semestre & 117 & 52 & 65 & 22.15 & 5.66 & 18 & 50 \\
\hline $4^{\circ}$ semestre & & 24 & 9 & 15 & 31 & 11.53 & 20 & 65 \\
\hline $6^{\circ}$ semestre & & 24 & 11 & 13 & 33.33 & 10.95 & 21 & 53 \\
\hline $8^{\circ}$ semestre & & 18 & 4 & 14 & 27.61 & 8.2 & 21 & 47 \\
\hline \multirow[t]{3}{*}{$9^{\circ}$ semestre } & & 6 & 2 & 4 & 35.66 & 5.34 & 24 & 40 \\
\hline & $9^{\circ}$ semestre & 87 & 41 & 46 & 26.31 & 6.45 & 21 & 49 \\
\hline & $10^{\circ}$ semestre & 15 & 9 & 6 & 34.53 & 12.28 & 21 & 63 \\
\hline
\end{tabular}

Fonte: Elaborado pelos autores

Conforme Tabela 1, participaram da pesquisa, da instituição 1, alunos do primeiro, segundo, quarto, sexto, oitavo e nono semestres, enquanto da instituição 2 , foram alunos do primeiro, segundo, nono e décimo semestres. Também participaram da pesquisa 23 professores das duas instituições de ensino, sendo 15 do gênero masculino e 8 do gênero feminino, conforme Tabela 2.

Tabela 2 - Características dos professores

\begin{tabular}{|c|c|c|c|c|c|c|c|c|c|c|}
\hline & \multicolumn{3}{|c|}{ sujeitos } & \multicolumn{4}{|c|}{ id ad e } & \multicolumn{3}{|c|}{ tempo d e experiên } \\
\hline & $\begin{array}{c}\mathbf{n} \\
\text { total } \\
\end{array}$ & homens & mulheres & Media & dp & min & máx & média & dp & $\min$ \\
\hline Instituiçãol & 9 & 3 & 6 & 45.11 & 6.82 & 35 & 54 & 6.1 & 4.2 & 1 \\
\hline Instituição 2 & 14 & 12 & 2 & 43.92 & 12.24 & 25 & 65 & 12.28 & 10.38 & 2 \\
\hline
\end{tabular}

Fonte: Elaborado pelos autores

Os dados foram tabulados no Excel ${ }^{\circledR}$ e, a partir deste, foram criadas as tabelas para descrever a amostra. Para análise inferencial dos dados foi utilizado software SPSS, versão 20.0 para realização da Análise Multivariada da Variância (MANOVA). 


\section{Resultados}

Este estudo identificou os estilos de aprendizagem no uso da tecnologia em estudantes e docentes do curso de Direito de duas Instituições. Os estudantes foram distribuídos por esta pesquisa em Grupo 1 e Grupo 2. O Grupo 1 era composto por alunos que estavam cursando do primeiro ao quinto semestre, enquanto no Grupo 2, os alunos cursavam o sexto ao décimo semestre. Esta pesquisa considerou as variáveis gênero e semestre em que o aluno cursava para análise estatística. Os professores do curso de Direito dessas instituições também foram avaliados, sendo que as variáveis consideradas foram gênero e instituição.

A Escala de Estilos de Aprendizagem em Situações de Uso de Tecnologia construída por Roza (2017), compreende os seguintes estilos: Estilo teórico (13 itens), Estilo pragmático (10 itens), Estilo de interação com o meio (8 itens) e Estilo de interação através do meio (8 itens).

Analisadas as respostas dos alunos das Instituições 1 e 2, tivemos os resultados apresentados na Tabela 3, que apresenta as pontuações mínima e máxima, as médias e os valores de desvio padrão por grupo e por gênero. Visualiza-se que os estudantes do Grupo 2 pontuaram, em média, mais que os estudantes do Grupo 1, em todos os estilos. Já quanto ao gênero, seguindo a análise por Grupos, embora os valores estejam muito próximos, com relação aos alunos do grupo da primeira metade do curso (Grupo 1), as mulheres pontuaram mais que os homens nos estilos pragmático e interação com o meio, enquanto os homens, consequentemente, pontuaram mais nos estilos teórico e através do meio. Já no Grupo 2 (alunos da segunda metade do curso), as mulheres pontuaram mais em todos os estilos do que os homens. Em uma análise geral, as pontuações médias dos dois grupos analisados, em cada um dos quatro estilos de aprendizagem, foram próximas, não havendo grandes diferenças entre elas. Da mesma forma com relação ao gênero, com pontuações próximas para os gêneros masculino e feminino, quando comparados em um mesmo estilo. 
Tabela 3 - Estilos de aprendizagem dos estudantes por gênero e grupo

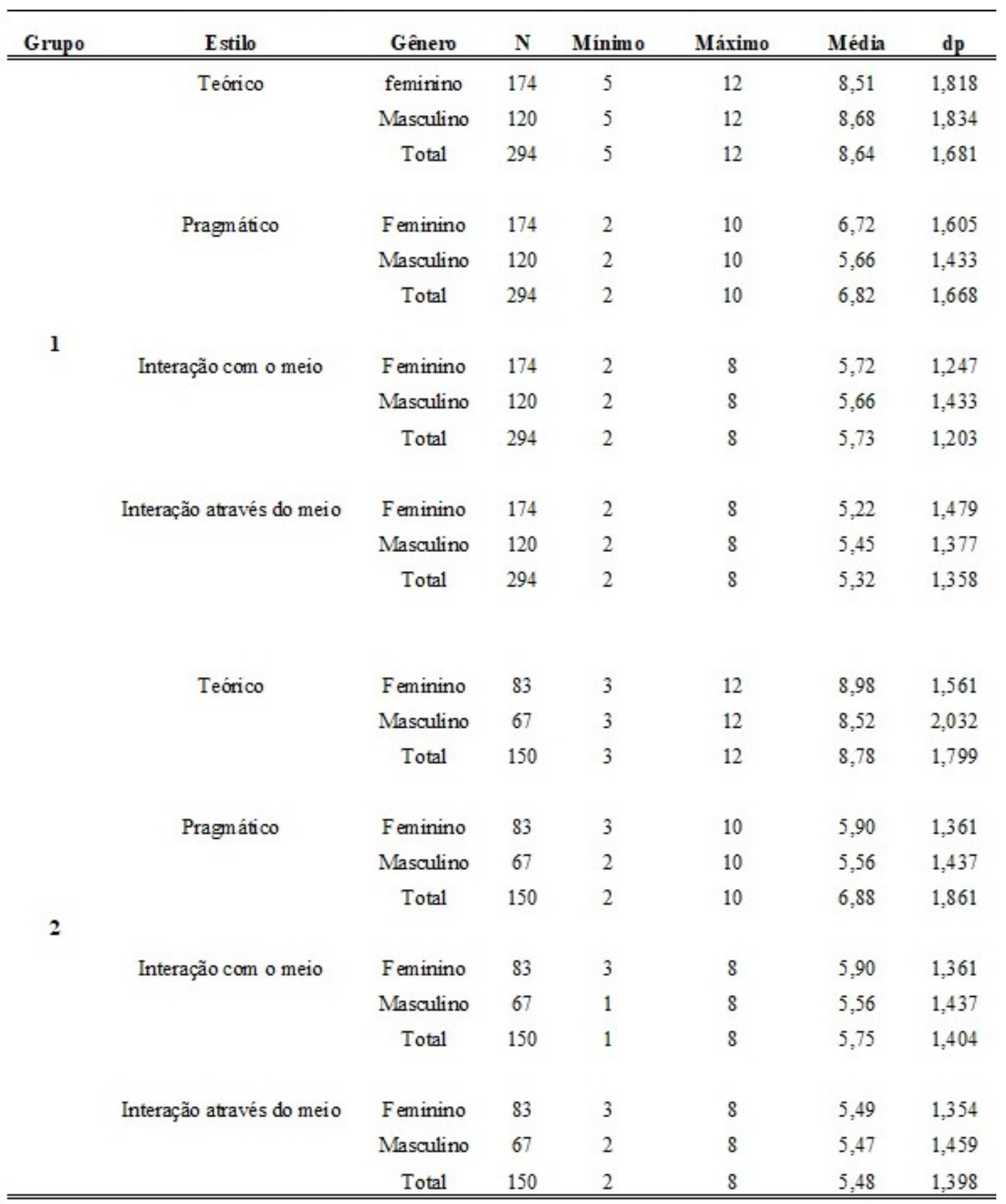

Fonte: Elaborado pelos autores

Já com relação aos estilos de aprendizagem dos professores, os docentes da instituição 1 pontuaram, em média, mais que os professores da instituição 2 em todos os estilos (tabela 4). Quanto ao gênero, seguindo a análise por instituição, na Instituição 1 os homens obtiveram as maiores médias nos estilos teórico, pragmático e de interação com o meio, enquanto os professores do gênero feminino tiveram a maior média somente no estilo de interação através 
do meio. Já na Instituição 2, os professores do gênero feminino obtiveram as maiores médias em todos os estilos, com uma diferença maior no estilo Interação através do meio (mulheres = 4.5 e homens $=1.08)$.

Tabela 4 - Estilos de aprendizagem dos professores por gênero e instituição

\begin{tabular}{ccccccc}
\hline Instituição & Estilo & Gênero & N & Mínimo & Máximo & Média \\
\hline \hline Teórico & feminino & 6 & 3 & 12 & 7,66 \\
& masculino & 3 & 8 & 13 & 10 \\
& total & 9 & 3 & 13 & 8,44 \\
& & & & & \\
& Pragmático & feminino & 6 & 7 & 9 & 8,33 \\
& masculino & 3 & 9 & 10 & 9,66 \\
& total & 9 & 7 & 10 & 8,77
\end{tabular}

1

$\begin{array}{cccccc}\text { Interação com o meio } & \text { feminino } & 6 & 1 & 8 & 5,66 \\ & \text { masculino } & 3 & 5 & 7 & 6 \\ \text { total } & 9 & 1 & 8 & 5,77\end{array}$

Interação através do meio

$\begin{array}{clllc}\text { feminino } & 6 & 3 & 6 & 4,66 \\ \text { masculino } & 3 & 2 & 6 & 4 \\ \text { total } & 9 & 3 & 6 & 4,44\end{array}$

$\begin{array}{cccccc}\text { Teórico } & \text { feminino } & 2 & 9 & 9 & 9 \\ \text { masculino } & 12 & 5 & 12 & 7,75 \\ \text { total } & 14 & 5 & 12 & 7,92\end{array}$

$\begin{array}{cccccc}\text { Pragmático } & \text { feminino } & 2 & 6 & 9 & 7,5 \\ & \text { masculino } & 12 & 1 & 10 & 6,5 \\ & \text { total } & 14 & 1 & 10 & 6,64\end{array}$

2

$\begin{array}{cccccc}\text { Interação com o meio } & \text { feminino } & 2 & 5 & 6 & 5,5 \\ & \text { masculino } & 12 & 1 & 8 & 4,41 \\ & \text { total } & 14 & 1 & 8 & 4,57\end{array}$

Interação através do meio

$\begin{array}{ccccc}\text { feminino } & 2 & 3 & 6 & 4,5 \\ \text { masculino } & 12 & 0 & 3 & 1,08 \\ \text { total } & 14 & 0 & 6 & 1,57\end{array}$

Fonte: Elaborado pelos autores

RIAEE - Revista Ibero-Americana de Estudos em Educação, Araraquara, v. 16, n. 3, p. 1823-1837, jul./set. 2021. e-ISSN: 1982-5587 
Objetivando verificar possíveis influências das variáveis gênero e grupo e de suas interações nos estilos de aprendizagem, foi feita a Análise Multivariada da Variância (MANOVA). Segundo os resultados mostrados na Tabela 5, não houve diferenças estatisticamente significantes de estilos de aprendizagem em relação às variáveis gênero e grupo, tampouco em relação à interação entre essas variáveis.

Tabela 5 - Análise Multivariada da Variância por gênero e por grupos

\begin{tabular}{ccccc}
\hline Variável & Estilo & Quadrado Médio & F & Sig. \\
\hline \multirow{3}{*}{ gênero } & Teórico & 3.085 & 0.929 & 0.336 \\
& pragmático & 0.028 & 0.009 & 0.925 \\
& com o meio & 5.709 & 3.122 & 0.078 \\
& através do meio & 0.415 & 0.202 & 0.653 \\
& & & & \\
grupos & Teórico & 3.775 & 1.137 & 0.287 \\
& pragmático & 1.466 & 0.472 & 0.493 \\
& como meio & 0.275 & 0.150 & 0.699 \\
& através do meio & 2.768 & 1.351 & 0.246 \\
& & & & \\
& Térico & 3.894 & 1.174 & 0.279 \\
gênerox grupos & pragmático & 0.194 & 0.062 & 0.803 \\
& como meio & 0.420 & 0.229 & 0.633 \\
& através do meio & 0.188 & 0.091 & 0.762 \\
& & & & \\
\hline
\end{tabular}

Fonte: Elaborado pelos autores

Segundo a Tabela 5, não houve diferenças estatisticamente significantes de estilos de aprendizagem em relação às variáveis gênero e grupo, tampouco em relação à interação entre essas variáveis.

\section{Considerações finais}

Esta pesquisa teve como objetivo identificar os estilos de aprendizagem no uso da tecnologia em alunos e docentes de curso de Direito e as influências das variáveis gênero e fase do estudante sobre estes estilos dos alunos, além de apresentar os pressupostos teóricos dos estilos de aprendizagem e dos estilos de uso da tecnologia. 
Diante das mudanças trazidas pela atual sociedade, denominada de sociedade da informação, decorrentes do desenvolvimento das tecnologias digitais, influenciando todos os setores da sociedade, dentre eles o educacional, torna-se necessário investigar sobre os estilos de aprendizagem no uso das tecnologias da informação e comunicação.

Cada indivíduo tem sua maneira de interagir com as situações que surgem no ambiente de aprendizagem, denominada de estilos de aprendizagem, sendo que a inserção das tecnologias da informação e comunicação na vida das pessoas, interferindo no modo de adquirir o conhecimento, acabará gerando também preferências de uso dos recursos tecnológicos.

Ocorre que a inserção de ambientes de aprendizagem informatizados irá exigir do professor um esforço diferenciado (BITTAR, 2000). A formação de professores, segundo experiências realizadas na escola com a informática, tem-se mostrado fundamental, exigindo uma abordagem bem diferente, uma vez que a implantação da informática no campo educacional abrange muito mais do que o mero conhecimento do professor sobre computadores ou metodologias para seu uso em uma disciplina (VALENTE; ALMEIDA, 1997).

Nesse sentido, segundo Valente e Almeida (1997), a proposta de mudança pedagógica através da introdução da informática na educação, conforme consta no Programa Brasileiro de Informática no Brasil, não visa criar condições para o professor dominar o computador ou o software, mas auxiliá-lo a desenvolver conhecimento sobre o conteúdo a ser ensinado e sobre como o computador pode ser utilizado no desenvolvimento desse conteúdo.

Percebe-se, portanto, na atividade docente, a importância da forma como o conhecimento irá chegar, de forma correta, aos discentes, através da prática pedagógica, ou seja, de que maneira o professor exercerá o papel de mediador do processo de ensino e aprendizagem (FIGUEIREDO; NOBRE; PASSOS, 2015).

Com relação aos estilos de aprendizagem, verificou-se nesta pesquisa que a compatibilidade entre os estilos do professor e dos alunos poderá ser um grande aliado do professor, trazendo muitos benefícios para o processo de ensino e aprendizagem. A pesquisa bibliográfica, através do banco de dados, também apontou a escassez de pesquisas sobre o uso das tecnologias digitais em cursos de Direito, sendo que, quanto ao tema estilos de aprendizagem no uso da tecnologia de alunos e docentes do curso de Direito, nenhum estudo foi encontrado. 


\section{REFERÊNCIAS}

ALONSO, C. M.; GALLEGO, D. Aprendizaje y ordenador. Madrid: Dykinson, 2000.

ALONSO, C. M.; GALLEGO, D. J.; HONEY, P. Los estilos de aprendizaje: procedimientos de diagnóstico y mejora. Madrid: Mensajero, 2002. Disponível em:

https://www.researchgate.net/publication/311452891_Los_Estilos_de_Aprendizaje_Procedim ientos_de_diagnostico_y_mejora. Acesso em: 20 out. $201 \overline{7}$.

ALONSO, C. M.; GALLEGO, D. J.; HONEY, P. Los estilos de aprendizaje: procedimentos de diagnóstico y mejora. 7. ed. Bilbao: Ediciones Mensajero, 1997.

AMARAL, S. F.; BARROS, D. M. V. Estilos de aprendizagem no contexto educativo de uso das tecnologias digitais interativas. In: SIMPÓSIO INTERNACIONAL SOBRE NOVAS COMPETÊNCIAS EM TECNOLOGIAS DIGITAIS INTERATIVAS NA EDUCAÇÃO, 1., 2007, São José dos Campos. Anais [...]. São José dos Campos, SP, 2007.

BARROS, D. M. V. Estilos de uso do espaço virtual: como se aprende e se ensina no virtual? Inter-Ação: Rev. Fac. Educ., v. 34, n. 1, p. 51-74, 2009.

BELLONI, M. L. Ensaio sobre a Educação a Distância no Brasil. Educação \& Sociedade, ano XXIII, n. 78, p. 117-142, 2002. Disponível em:

http://www.scielo.br/pdf/es/v23n78/a08v2378.pdf. Acesso em: 05 dez. 2019.

BITTAR, M. Informática na educação e formação de professores no Brasil, Série-Estudos, Campo Grande, n. 10, p. 91-106, 2000.

BUTSZKE M. A.; ALBERTON, A. Estilos de aprendizagem e jogos de empresa: a percepção discente sobre estratégia de ensino e ambiente de aprendizagem, REGE- Revista de Gestão, v. 24, p. 72-84, 2017.

CINTRA, C. C. S.; CAMURÇA, E. E. P.; REIS, U. L. S. O uso de novas tecnologias de informação e comunicação nas salas e aula da faculdade de Direito da Universidade Federal do Ceará: ferramentas agregadoras ou disruptivas do processo de ensino- aprendizagem?

Revista do Programa de Pós-Graduação em Direito da UFC, v. 37, n. 2, p. 405-422, 2017.

COUTINHO, C.; LISBÔA, E. Sociedade da informação, do conhecimento e da

aprendizagem: desafios para educação no século XXI. Revista de Educação, v. 28, n. 1, p. 5$22,2011$.

ESTEVÃO, R. B.; PASSOS, G. O. O programa nacional de tecnologia educacional (PROINFO) no contexto da descentralização da política educacional brasileira. Holos, ano 31, v. 1, p. 199-210, 2015.

FIGUEIREDO, G. L. R.; NOBRE, I. A. M.; PASSOS, M. L. S. Tecnologias computacionais na educação: desafios na prática docente. In: WORKSHOP DE INFORMÁTICA NA ESCOLA (WIE), 21., 2015. Anais [...]. Porto Alegre, RS, 2015. 
GOES, F. S. N. et al. Tecnologias educacionais digitais para educação profissional de nível médio de enfermagem. Revista eletrônica de enfermagem, v. 16, n. 2, p. 453-61, abr./jun. 2014.

GONÇALVES, A. et al. Avanço na modelagem automática e dinâmica de estilos de aprendizagem de estudantes em sistemas adaptativos e inteligentes para educação: uma análise experimental. In: CONGRESSO BRASILEIRO DE INFORMÁTICA NA EDUCAÇÃO, 5.; SIMPÓSIO BRASILEIRO DE INFORMÁTICA NA EDUCAÇÃO, 18. 2016. Anais [...]. Porto Alegre, RS: CBIE, SBIE, 2016.

KENSKI, V. Aprendizagem mediada pela tecnologia. Revista diálogo educacional, v. 4, n. 10, p. 1-10, 2003. Disponível em: http://www2.pucpr.br/reol/pb/index.php/dialogo? dd1=786\&dd99=view\&dd98=pb. Acesso em: 05 dez. 2019.

LACERDA, A. L.; SILVA. T. Materiais e estratégias didáticas em ambiente virtual de aprendizagem. Rev. bras. Estud. pedagog., Brasília, v. 96, n. 243, p. 321-342, 2015.

\section{LÂNGARO, A. G. Tecnologia e práticas pedagógicas - movimentos e vicissitudes na} busca da constituição de uma comunidade de aprendizagem. 2003. 145 f. Dissertação (Mestrado em Psicologia Social e Institucional) - Universidade Federal do Rio Grande do Sul, Porto Alegre, 2003.

LEMOS, E. C.; AMARAL, L. A. M.; OLIVEIRA, L. R. M. Utilização de estilos de aprendizagem no desenvolvimento de ambientes virtuais voltados à educação à distância. Revistas de Estilo de Aprendizagem, v. 8, n. 15, 2015. Disponível em: http://learningstyles.uvu.edu/index.php/jls/article/view/229/187. Acesso em: 11 out. 2017.

LIMA FILHO, N.; BEZERRA, E. S.; SILVA, T. B. S. Estilo de aprendizagem dos alunos do curso de Ciências Contábeis. Revista Gestão Universitária na América Latina-GUAL, v. 9, n. 2, p. 95-112, 2016.

MARQUES, C. A. M. O ensino jurídico e as novas tecnologias de informação e comunicação. Revista de Educação, v. 13, n. 16, p. 199-214, 2010.

MEURER, A. M. et al Estilos de aprendizagem em rendimento acadêmico na universidade. REICE. Revista Iberoamericana sobre Calidad, Eficacia y Cambio en educación, v. 16, n. 4, 2018. DOI: 10.15366/reice2018.16.4.002

MUNFORD, A.; HONEY, P. Questions and answers on learnig styles questionnaire. Industrial and Commercial Training, v. 24, n. 7, p. 10-13, 1992.

PÚBLIO JR, C. O docente e o uso das tecnologias no processo de ensinar e aprender. RIAEE - Revista Ibero-Americana de Estudos em Educação, Araraquara, v. 13, n. 03, p. 10921105, jul./set. 2018. e-ISSN: 1982-5587. DOI: 10.21723/riaee.v13.n3.2018.11190

RIEDNER, D. D. T.; PISCHETOLA, M. Tecnologias Digitais no Ensino Superior: uma possibilidade de inovação das práticas? Educação, Formação \& Tecnologias, v. 9, n. 2, p. 37-55, 2016. 
ROZA, R. H. Estilos de aprendizagem e o uso das tecnologias da informação e comunicação. 2017. Tese (Doutorado em Psicologia) - Centro de Ciências da Vida, Pontifícia Universidade Católica de Campinas, Campinas, 2017.

SALVADOR, P. T. C. O. et al. Uso e desenvolvimento de tecnologias para o ensino apresentados em pesquisas de enfermagem. Revista da Rede de Enfermagem do Nordeste, v. 16, n. 3, p. 442- 450, 2015.

SILVA, D. M. et al. Estilos de aprendizagem e desempenho acadêmico na Educação a Distância: uma investigação em cursos de especialização. Revista Brasileira de Gestão e Negócios, São Paulo, v. 17, n. 57, p. 1300-1316, 2015.

SILVA, G. O. L. Construção e evidências de validade e precisão de escala de estilos de aprendizagem em universitários. 2011. Tese (Doutorado em Psicologia) - Centro de Ciências da Vida, Pontifícia Universidade Católica de Campinas, Campinas, 2011.

SILVA, J. B. O contributo das tecnologias digitais para o ensino híbrido: o rompimento das fronteiras espaço-temporais historicamente estabelecidas e suas implicações no ensino", ARTEFACTUM - Revista de Estudos em Linguagem e Tecnologia, ano IX, n. 02, 2017.

SILVEIRA, D. S.; NOVELLO, T. P.; LAURINO, D. P. Tecnologias digitais na Educação superior: compreensões acerca da formação permanente de professores em uma rede de conversação. Revista Thema, v. 15, n. 3, 2018.

SOUZA, A. M. As tecnologias da informação e da comunicação (TIC) na educação para todos. Educ. Foco, Juiz de Fora, Edição Especial, p. 349-366, fev. 2015.

VALENTE, J. A.; ALMEIDA, F. J. Visão analítica da informática na educação no Brasil: a questão da formação do professor, Revista Brasileira de Informática na Educação, n. 1, 1997.

VIANA, H. B.; SILVA, G. O. L.; DINIGRE, W. Experiências de formação e atuação docente com utilização de tecnologias. Revista Tecnologia Educacional, v.17, n. 222, p. 27-37, 2018 . 


\section{Como referenciar este artigo}

VIANA, H. B.; RAMOS, M. L.; ROZA, R. H. Estilos de aprendizagem e tecnologias da informação e comunicação no curso de direito. Revista Ibero-Americana de Estudos em Educação, Araraquara, v. 16, n. 3, p. 1823-1837, jul./set. 2021. e-ISSN: 1982-5587. DOI: https://doi.org/10.21723/riaee.v16i3.13453

Submetido em: 18/03/2020

Revisões requeridas em: 20/07/2020

Aprovado em: 15/08/2020

Publicado em: 01/07/2021 